\title{
Acquiring and maintaining a normal oral microbiome: current perspective
}

\author{
Egija Zaura $^{1}{ }^{*}$, Elena A. Nicu ${ }^{2}$, Bastiaan P. Krom ${ }^{1}$ and Bart J. F. Keijser ${ }^{3,4}$ \\ ${ }^{1}$ Department of Preventive Dentistry, Academic Centre for Dentistry Amsterdam, Amsterdam, Netherlands \\ 2 Department of Periodontology, Academic Centre for Dentistry Amsterdam, Amsterdam, Netherlands \\ ${ }^{3}$ Microbiology and Systems Biology, TNO Earth, Environmental and Life Sciences, Zeist, Netherlands \\ ${ }^{4}$ Top Institute Food and Nutrition, Wageningen, Netherlands
}

\section{Edited by:}

Alex Mira, Center for Advanced

Research in Public Health, Spain

Reviewed by:

Sung Ouk Kim, University of

Western Ontario, Canada

Elizabeth B. Norton, Tulane

University, USA

*Correspondence:

Egija Zaura, Department of

Preventive Dentistry, Academic

Centre for Dentistry Amsterdam,

Gustav Mahlerlaan 3004,

1081LA Amsterdam, Netherlands

e-mail: e.zaura@acta.nl
The oral microbiota survives daily physical and chemical perturbations from the intake of food and personal hygiene measures, resulting in a long-term stable microbiome. Biological properties that confer stability in the microbiome are important for the prevention of dysbiosis-a microbial shift toward a disease, e.g., periodontitis or caries. Although processes that underlie oral diseases have been studied extensively, processes involved in maintaining of a normal, healthy microbiome are poorly understood. In this review we present our hypothesis on how a healthy oral microbiome is acquired and maintained. We introduce our view on the prenatal development of tolerance for the normal oral microbiome: we propose that development of fetal tolerance toward the microbiome of the mother during pregnancy is the major factor for a successful acquisition of a normal microbiome. We describe the processes that influence the establishment of such microbiome, followed by our perspective on the process of sustaining a healthy oral microbiome. We divide microbiome-maintenance factors into host-derived and microbe-derived, while focusing on the host. Finally, we highlight the need and directions for future research.

Keywords: oral microbiome, placenta, tolerance, mucosal immunity, stability, colonization resistance

\section{INTRODUCTION}

The oral microbiota needs to cope with daily physical and chemical perturbations from the intake of food and personal hygiene measures. These include fluctuations in temperature, $\mathrm{pH}$, antimicrobial and dietary components, and mechanical sheer forces from brushing and mastication. Intriguingly, a long-term stable microbiome is maintained in the oral cavity, as demonstrated by Rasiah and colleagues by following an individual saliva donor over a period of 7 years (Rasiah et al., 2005). Recent data from the NIH Human Microbiome Project (HMP) revealed that the oral microbiome has the largest core of commonly shared microbes among unrelated individuals compared to other habitats such as gut or skin (Costello et al., 2009; Li et al., 2013; Zhou et al., 2013).

A key question is what governs the stability of the oral microbiome in health? Biological properties that confer stability in the microbiome are important for the prevention of dysbiosis-a microbial shift toward a disease, e.g., periodontitis or caries and sustaining general health (for review see Wade, 2013). Although processes that underlie oral diseases have been studied extensively (Bartold and Van Dyke, 2013; Bradshaw and Lynch, 2013; Nyvad et al., 2013; Belibasakis, 2014), processes behind the maintaining of a normal microbiome are poorly understood. In this review we present our hypothesis on how a healthy oral microbiome is acquired and maintained. We start by defining what constitutes a normal oral microbiome. Then we present our hypothesis on the mechanisms for acquiring a stable normal microbiome. Finally, we discuss some of the mechanisms involved in maintaining such a microbiome and highlight the directions for possible further research.

\section{WHAT CONSTITUTES NORMAL ORAL MICROBIOME?}

The human oral cavity is colonized by a wide range of microorganisms. Besides bacteria and fungi, Archaea, viruses and protozoa form a part of a normal microbiome (Wade, 2013). Current reports on a normal oral microbiome however are limited to the "bacteriome" (subsequently referred to as "microbiome") and very limited reports on the mycobiomefungal microbiome (Ghannoum et al., 2010; Dupuy et al., 2014; Mukherjee et al., 2014). Current knowledge on the role of fungi as part of a healthy oral microbiome has been recently reviewed and is therefore not further discussed here (Krom et al., 2014). The microbiome has been studied in great detail and phylogenetic information of oral bacteria is gathered in databases dedicated to oral cavity (Palmer, 2014). The HMP assessed microbiome composition of nine intraoral sites (buccal mucosa, hard palate, keratinized gingiva, palatine tonsils, saliva, sub- and supragingival plaque, throat and tongue dorsum) from about 200 subjects and found 185-355 genera, belonging to 13-19 bacterial phyla (Zhou et al., 2013). An individual sample (i.e., from a single site of a single volunteer) contained sequences classified to $20-50$ genera from 6 to 9 phyla. Table 1 summarizes the high abundance 
Table 1 | The core bacterial taxa in the oral cavity from over 200 healthy individuals participating in HMP (Li et al., 2013).

\begin{tabular}{|c|c|c|c|}
\hline Sample type & $\begin{array}{l}\text { High abundance core genera in }>75 \% \\
\text { samples at }>10 \% \text { abundance }\end{array}$ & $\begin{array}{l}\text { Other major core genera in }>80 \% \text { samples at } \\
>1 \% \text { abundance }\end{array}$ & $\begin{array}{l}\text { Minor core genera in }>50 \% \\
\text { samples }\end{array}$ \\
\hline Buccal mucosa & Streptococcus (2) & $\begin{array}{l}\text { Uncl. Pasteurellaceae }(16,19) \\
\text { Gemella (11) }\end{array}$ & $\begin{array}{l}\text { Atopobium } \\
\text { Uncl. Prevotellaceae } \\
\text { Uncl. Bacilli } \\
\text { Catonella }\end{array}$ \\
\hline
\end{tabular}

\begin{tabular}{ll}
\hline Keratinized gingiva & Streptococcus (2) \\
& Uncl. Pasteurellaceae (19)
\end{tabular}

\begin{tabular}{lll}
\hline Palatine tonsils & Streptococcus (2, 6) & Mogibacterium \\
Uncl. Firmicutes \\
& Veillonella (4) & Prevotella (10) \\
& Fusobacterium (9) \\
& Uncl. Pasteurellaceae (16)
\end{tabular}

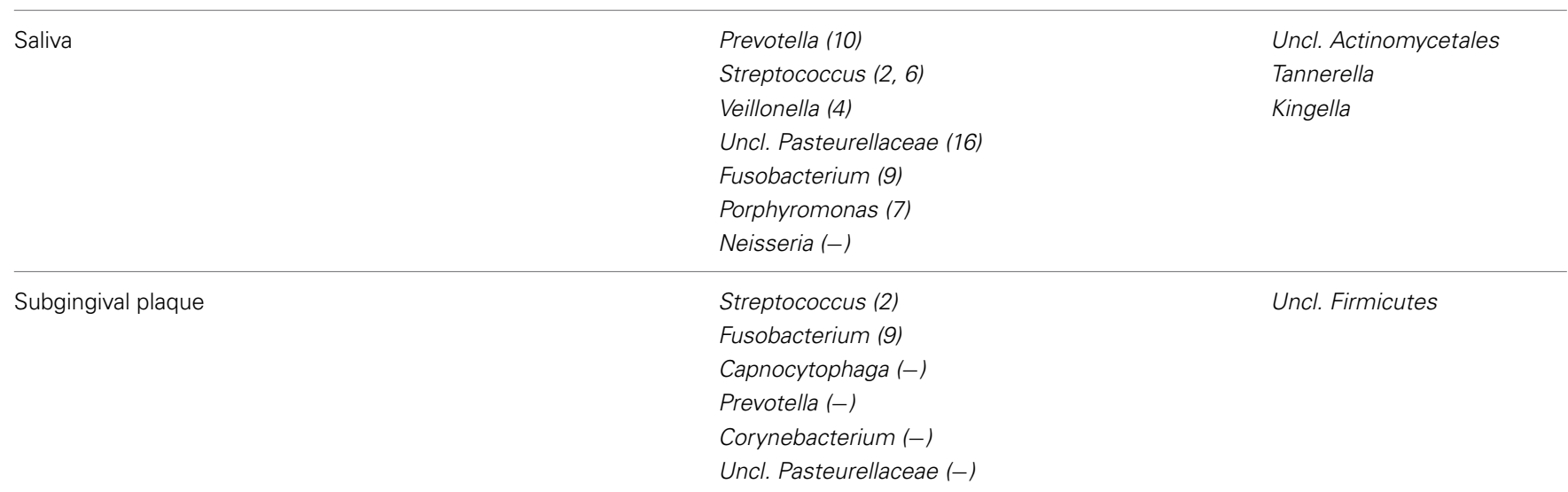

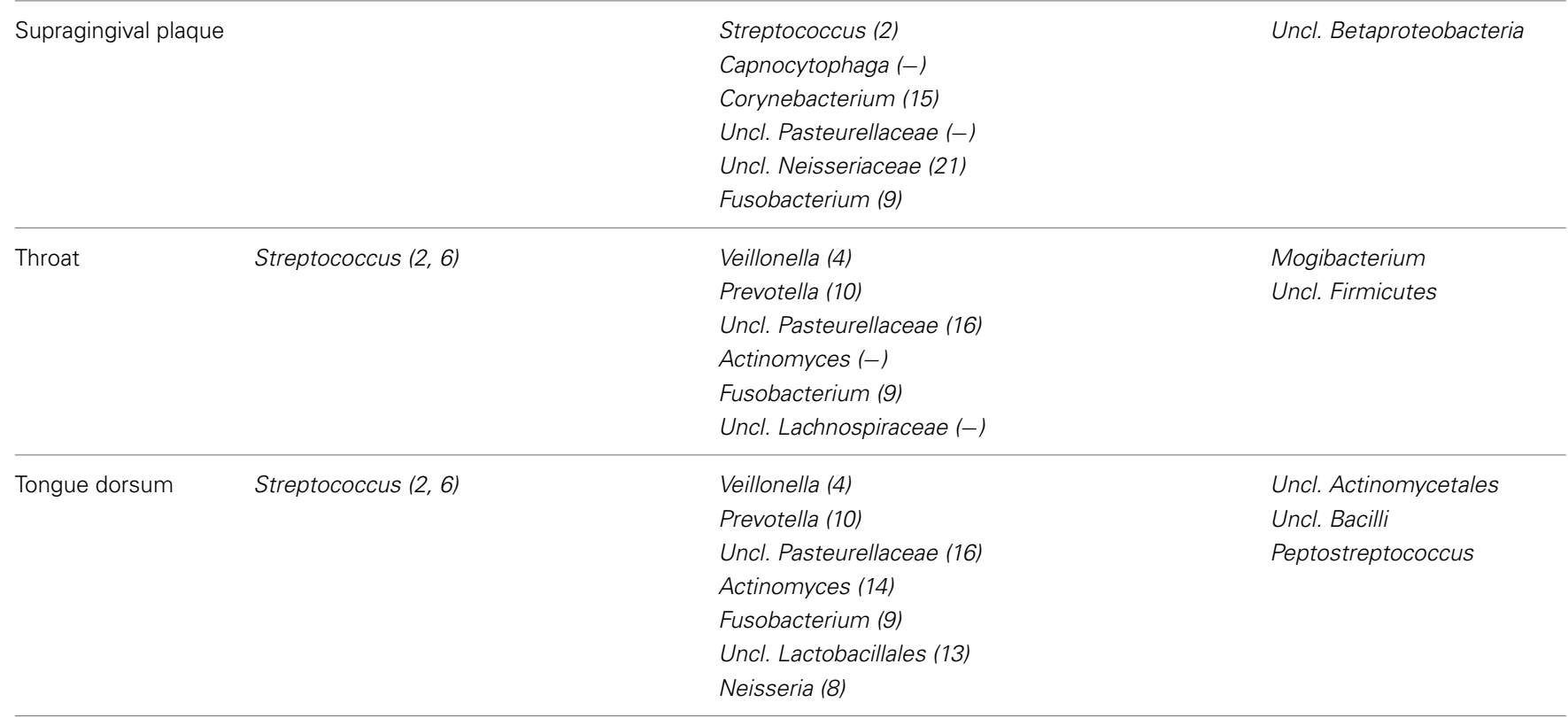

In the parentheses-the corresponding OTU in the genus or family. Uncl, unclassified. 
core genera (defined as genera present at $>10 \%$ abundance and at $>75 \%$ ubiquity) and other major core genera ( $>1 \%$ abundance at $>80 \%$ ubiquity), as well as operational taxonomic units (OTUs, $16 \mathrm{~S}$ rRNA gene variable v3-v5 region sequences, clustered at 97\% similarity) in these oral samples (Li et al., 2013). A single OTU, Streptococcus OTU\#2, dominated nearly all oral mucosal sites of this large cohort. The reads of this OTU were obtained from Kelvin Li (personal communication) and blasted (NCBI web site, megablast against $16 \mathrm{~S}$ ribosomal RNA sequences, default parameters) for finer taxonomic classification. The most abundant read of this OTU was identical over its entire length to $16 \mathrm{~S}$ rRNA gene sequences of Streptococcus oralis, Streptococcus mitis and Streptococcus peroris.

The microbiome has evolved through hundreds of thousands of years of co-habiting into a microbe-human symbiosis with mutual benefits (Hooper and Gordon, 2001; Clemente et al., 2012). The oral microbiome in newborns has been shown to seed the gut microbiome that first resembles that of the oral cavity and diverges in 2 weeks time to gut-specific communities (Costello et al., 2013). Recently a significant association between distinct microbial community types of stool and oral samples from HMP study adult population was demonstrated (Ding and Schloss, 2014). Based on current knowledge it is apparent that the acquisition of such normal, beneficiary microbiome by newborns is an essential process. Infants are colonized rapidly after birth by bacteria present in their direct environment, through bacterial transfer from their mother but also from other sources. How does a newborn discriminate between "friend and foe" among the diverse microbes in its postnatal environment? If any randomly trespassing microbe would permanently colonize oral cavity of the newborn, there would be no core microbiome (Table 1) and the benefit of the long-lasting co-evolution would be lost. We suggest that development of fetal tolerance toward the microbiome of the mother during pregnancy is the major factor for a successful acquisition of a normal microbiome. We have summarized our hypothesis in the text below and in Figure 1.

\section{DEVELOPMENT OF PRENATAL TOLERANCE TO MOTHER'S ORAL MICROBIOME}

Although the first encounter of a newborn with microbiota is considered to be postnatal, there is clinical evidence for microbial presence in placenta, umbilical cord blood, amniotic fluid, and meconium in full-term pregnancies without overt infection (Bearfield et al., 2002; Jiménez et al., 2005, 2008; Stout et al., 2013; Aagaard et al., 2014). Experimental intravenous infection of pregnant mice with pooled salivary or plaque microbes resulted in colonization of placenta by selected oral microorganisms (Fardini et al., 2010). Recent comparison of sequencing results from 320 placental microbiomes with the HMP dataset showed that the placental microbiome does not resemble vaginal or gut microbiomes as previously thought, but at least at a phylum level is most similar to normal oral microbiome, especially that from tongue and tonsils (Aagaard et al., 2014). These studies may suggest that the placental microbiome has a biological function. We propose that during pregnancy the placenta becomes an antigencollecting site for the fetal immune system to be "trained" in antigen tolerance. We suggest a hematogenous route for indigenous microbes to placenta during pregnancy. Pregnant women have increased gingival bleeding, diagnosed as pregnancy gingivitis (Niederman, 2013). We propose a new role for the increased gingival bleeding: by opening the vascular bed, oral bacteria from the mother become available in blood and thus gain access to the placenta. Jeurink and colleagues have introduced a similar mechanism for the formation of the breast milk microbiome. This involves immune cell education by the pregnancy hormone progesterone resulting in transportation of bacteria from the mother to her mammal glands (Jeurink et al., 2013). A similar process could be responsible for transporting bacteria to the placenta. Microbial cells are trapped in placental tissue to be presented to the fetal immune system. In the prenatal period, fetal antigen presenting cells (APCs) interact with the mother's microbial antigens and return to fetal peripheral lymphoid organs. The human fetus harbors large numbers of peripheral regulatory $\mathrm{T}$ cells (Tregs) with immunosuppressive activity (Takahata et al., 2004). Fetal Tregs can be retrieved also from umbilical cord blood offering a promising perspective for transplantation medicine, while newborns have been shown to have higher proportions of thymically derived Tregs than adults (Rabe et al., 2014). The fetal Tregs are preventing undesirable alloreactivity to maternal derivatives during the pregnancy (Takahata et al., 2004). As a result, the fetus develops prenatal tolerance to the mother's microbiome and regards it as "safe" during postnatal encounters with these bacteria. There is experimental and clinical evidence for development of fetal antigen-specific tolerance: human fetal Tregs become functionally suppressive after stimulation with maternal alloantigens and persist at least until early adulthood (Mold et al., 2008). If and to what extent early tolerance of the fetus toward the oral bacterial species is key to the colonization of the mouth and gastro-intestinal tract of the newborn and its impact in health development in the earliest phases of life needs to be investigated.

\section{ACOUIRING THE ORAL MICROBIOME}

Vertical transmission from mother to child starts at birth. The delivery mode (vaginal or Caesarian) will to a large extent determine which microorganisms - vagina or skin-derived-will be encountered first by the newborn (Dominguez-Bello et al., 2010). This affects the diversity of the oral microbiome: vaginally born infants showed higher taxonomic diversity at 3 months of age (Lif Holgerson et al., 2011). Interestingly, the birth mode may have a lasting impact as infants born with Caesarian section acquired Streptococcus mutans almost 1 year earlier (at 17.1 months of age) than vaginally born infants (28.8 months) (Li et al., 2005).

The method of feeding (breast-feeding or infant formula) affects the infant's microbiome as well. Breast-fed infants of 3 months of age carried oral lactobacilli with antimicrobial properties not found in formula-fed infants (Holgerson et al., 2013; Romani Vestman et al., 2013). In addition to this vertical transmission mechanism, horizontal transmission of oral microbiota, e.g., among siblings and other people sharing the same environment, contributes to oral microbiome diversity (Baca et al., 2012; Stahringer et al., 2012).

Once established, the microbiome should be sustained. Below we summarize our perspective on this process by dividing it into host-derived and microbe-derived microbiome maintenance 


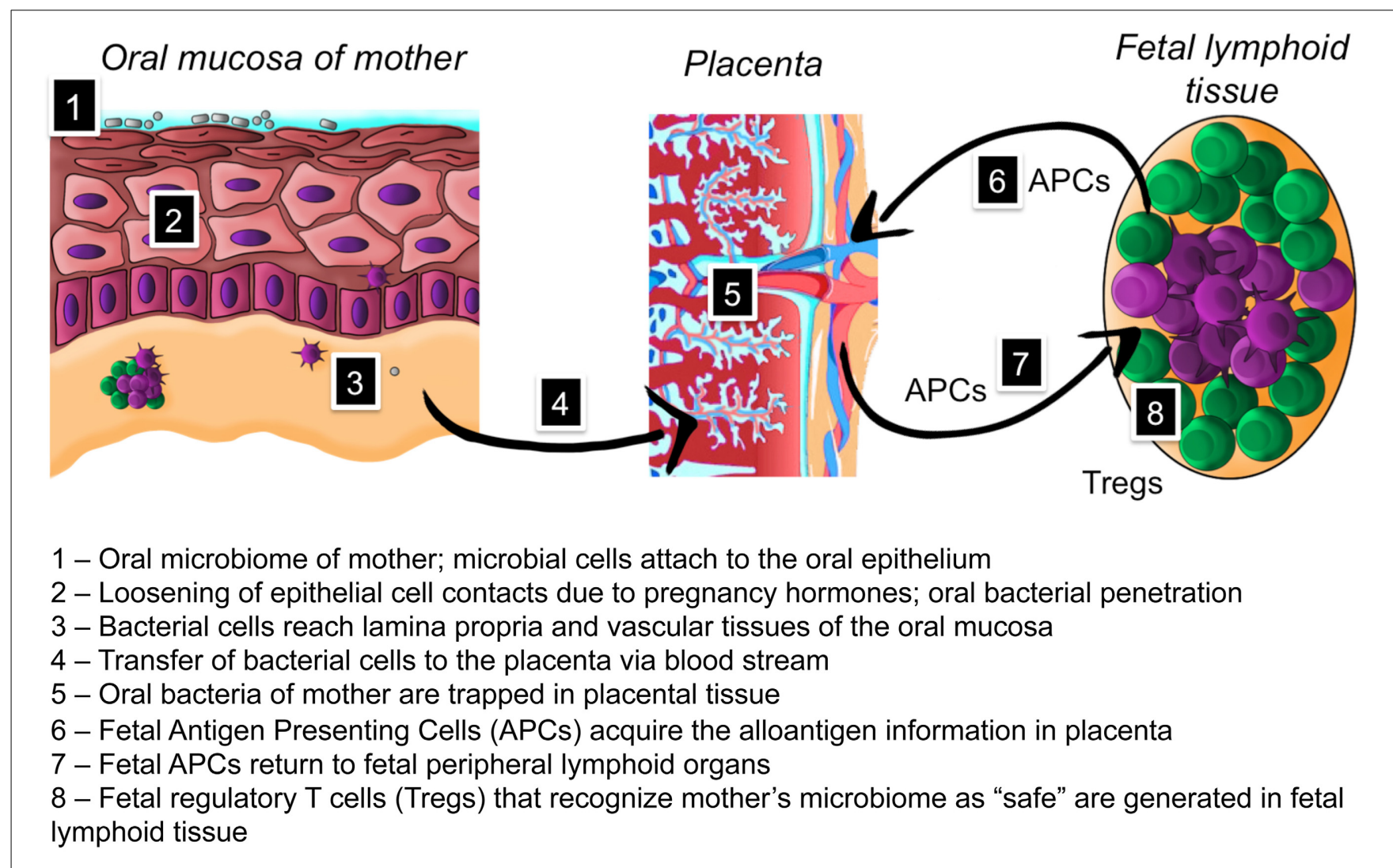

FIGURE 1 | Hypothesis on the role of placental microbiome in the development of fetal tolerance toward oral microbiome of mother.

factors. Since microbial factors have been extensively addressed elsewhere (Kuramitsu et al., 2007; Wright et al., 2013), we focus here mainly on the role of host factors.

\section{HOST-DERIVED MICROBIOME MAINTENANCE FACTORS}

The interactions between the microbiome and the host are bidirectional. In the absence of timely and adequate stimulation by the microbiome, germ-free mice show extensive deficiencies in intestinal immune system development, with reduced lymphoid tissue and fewer lymphocytes (Macpherson and Harris, 2004). In humans, early-life exposure to microbiota is protective for immune-mediated diseases such as asthma and inflammatory bowel disease (Olszak et al., 2012). The interplay between the microbiome and innate and adaptive immunity is site specific. The skin microbiome of mice is largely unaffected by genetic manipulation of innate or adaptive immunity, while mucosal sites-oral and gut microbiomes-are shaped by innate and adaptive immune responses (Scholz et al., 2014).

The human immune system develops in a continuing dialogue with the commensal microbial populations. One communication route between microbiota and host is via the host pattern recognition receptors (PRRs), with the Toll-like receptor (TLR) family playing a key role. In the gut, ligands binding to TLR-2 can favor Treg expansion (Sutmuller et al., 2006), while TLR-9-mediated recognition of DNA from gut flora is essential for effector T-cells to overcome Treg inhibition and mount an immune response
(Hall et al., 2008). Cells of the oral mucosa (keratinocytes, macrophages, dendritic cells (DCs), polymorphonuclear leukocytes and natural killer cells) express most of the TLRs (Feller et al., 2013), while altered expression patterns of TLRs have been found in oral pathology (Janardhanam et al., 2012).

Despite dense bacterial colonization, acute infections are rare in the oral mucosa, suggesting that this site is predominantly tolerogenic (Novak et al., 2008). Mucosal DCs are the arbiters of mucosal tolerance: on the one hand they are capable of mounting an effective defense against harmful pathogens, while on the other hand they inhibit immune reactions against antigens derived from commensal bacteria, preserving the health benefits acquired through thousands of years of co-evolution. DCs in the oral mucosal epithelium are of the Langerhans cell subtype and they employ complex regulatory mechanisms: deletion of T-cells via apoptosis, functional inactivation of T-cells, inhibition by co-inhibitory receptors, but also the development of antigen specific Tregs (Novak et al., 2008). Expression of LPS receptor CD14, TLR2, and TLR4 by DCs in the non-inflamed oral mucosa is crucial for the induction of tolerance. One of the mechanisms involved is the induction of Tregs characterized by interleukin (IL)-10 and transforming growth factor (TGF)$\beta$ secretion (Allam et al., 2008). In the gut, resident DCs are programmed by intestinal epithelial cells to suppress inflammation and promote immunological tolerance; in the case of a pathogen attack, "non-educated" DCs are recruited to initiate 
inflammation and a protective immune response against the invader (Iliev et al., 2007). A similar mechanism could also account for the oral mucosal tolerance. Indeed, a significant infiltration of pro-inflammatory plasmacytoid DCs is found in oral inflammation (Santoro et al., 2005).

Besides the PRRs, the host may also use chemical sensing to monitor microbial activity. Well known is epithelial signaling of short chain fatty acids involving dedicated G-coupled receptors (GPR41, GPR43) on gut epithelial cells (Layden et al., 2013). Recent studies suggest a direct link between secreted bacterial products and chemosensory activation mechanisms for mucosal clearance (Lee et al., 2014). Oral mucosa, but also airway epithelial cells and airway smooth muscle cells express bitter taste receptors (T2Rs) (Prince, 2012). The T2R38 receptor is activated directly by acyl-homoserine lactone (AHL) quorum sensing molecules produced by Pseudomonas aeruginosa and other Gram-negative bacteria and thus provide a mechanism for chemical sensing of bacterial colonization (Lee et al., 2012). Genetic differences in the T2R38 receptor, conferring an increased ability to perceive the bitter-tasting phenyl-thiocarbamide (supertaster phenotype), have been shown to underlie differences in the ability to signal presence and subsequent clearance of P.aeruginosa biofilm by respiratory epithelial cells (Lee et al., 2012). Intriguingly, specific alleles of the T2R38 bitter taste receptor have also been associated with a reduced risk for caries (Wendell et al., 2010). While this association was significant for the primary dentition, no significant associations could be identified for the mixed or permanent dentition groups. In addition, decreased taste sensitivity for another bitter-tasting chemical, 6n-propylthiouracil, was associated with increased risk for dental caries and higher mutans streptococci counts (Shetty et al., 2014). Although the ability to taste bitter components may influence dietary habits, it is tempting to suggest a mechanistic relationship between bitter taste and antimicrobial defense also in the oral cavity.

An important oral immunity factor is delivered via saliva and gingival crevicular fluid-the secretory immunoglobulin A (S-IgA). These antibodies limit and control microbial adhesion and colonization (Feller et al., 2013). IgA proteases that neutralize S-IgA are known virulence factors of human pathogens such as Neisseria meningitidis and Streptococcus pneumoniae. However, also commensal streptococci such as Streptococcus mitis 1, Streptococcus oralis and Streptococcus sanguinis are able to produce IgA proteases (Kilian et al., 1996). These commensal oral streptococci are primary colonizers and the major species colonizing infants and mucosal sites in adults (Marsh et al., 2009). Their ability to circumvent S-IgA guarantees survival in the oral cavity and underlines their long-lasting symbiotic co-evolution with the human host.

Salivary glycoproteins contain glycans that may act as decoys to prevent pathogens from adhering to epithelial cells, thereby influencing a healthy microbial homeostasis. This was shown in vitro on inhibition of adhesion of the fungus Candida albicans to epithelial cells (Everest-Dass et al., 2012). Other salivary proteins that influence the oral microbiome include lactoferrin, agglutinins, lysozyme, peroxidase, statherin, histatins, defensins, and mucins (for extensive review see Dodds et al., 2005). For instance, histatin 5 has candicidal activity and its concentration is reduced in saliva of elderly and HIV patients (Khan et al., 2013) two populations prone to candidiasis. Salivary flow rate as well as composition thus play key role in maintaining a healthy oral microbiome.

The impact of the immune system on oral health is most obvious once it becomes dysfunctional, as in hematopoietic stem cell transplant patients who have received immunosuppressive therapy. In these patients, the mucosal barrier is often damaged leading to severe mucositis with life-threatening viral and fungal infections (Petti et al., 2013), as well as oral infections by non-oral species (Soga et al., 2011; Diaz et al., 2013).

\section{MICROBE-DERIVED MICROBIOME MAINTENANCE FACTORS}

Co-evolution of the microbiome with the host has resulted in host-associated microbial communities that are equipped with mechanisms that allow them to prevent colonization and establishment of foreign microbes, so called "colonization resistance" (He et al., 2013). An extensive review on interspecies interactions within oral communities has been provided by Kuramitsu and colleagues. The authors distinguished five types of interaction between oral bacteria: competition for nutrients, synergy, antagonism, neutralization of virulence factors and interference in signaling mechanisms (Kuramitsu et al., 2007). Integrity of the microbial community is maintained by specific inter-microbial adhesion, cell signaling through cell-to-cell contact, metabolic interactions and quorum sensing (Wright et al., 2013). Recently a social structure in the murine oral community was reported where a concerted action by "sensor," "mediator" and "killer" bacteria in the community formed a pathway that prevented colonization by the non-oral species Escherichia coli (He et al., 2013).

Besides bacterial inter-species communication, also interkingdom communication plays a role in oral microbial ecosystem (Morales and Hogan, 2010; Jarosz et al., 2011). Bacteria produce a range of signaling molecules that affect $C$. albicans biofilm formation or morphogenesis, while C. albicans metabolites are known to influence bacterial growth (Wright et al., 2013). Such interactions, as well as interkingdom adhesion events, are also likely with the other members of the oral mycobiome (Krom et al., 2014).

\section{FUTURE RESEARCH DIRECTIONS}

A stable ecosystem is the result of complex interactions between all members of the system (Jenkinson, 2011). The entire oral microbiome of an individual, in addition to bacteria also fungi, viruses, Archaea and protozoa, has so far not been approached as an entity. The current knowledge is based on studying individual community components without the complexity of their mutual interactions. The first article on assessment of both, fungal and bacterial profiles in the same oral samples has just been published (Mukherjee et al., 2014), although focusing on diseased individuals, infected with HIV.

Microbiology of oral health has been neglected for decades. With the advent of health-related microbiome research grants such as HMP, an important turning point has been set, with a paradigm shift from disease as a starting point toward health as main interest. Since our current knowledge on the mechanisms 
of oral microbiome stability is only revealing the tip of the iceberg any initiatives similar to NIH HMP need to be encouraged. The Dutch Top Institute of Food and Nutrition (TIFN) (www.tifn. nl) has established public-private partnership that aims to identify the biological processes in the oral ecosystem responsible for maintaining oral health and to develop in vitro and in vivo technologies for the development of novel preventative strategies and to evaluate their efficacy. The project is based on the hypothesis that oral health reflects the ability of the oral ecosystem to adapt to and counteract perturbing stresses, where the oral ecosystem is defined as the oral microbiota, the saliva and host (mucosal) immunity.

The introduction of novel sequencing technologies has led to a significant advance in our knowledge of the oral microbiome in its broadest sense, and has revealed a stable commensal population, suggesting symbiotic beneficial relationship. However, the underlying principles of the beneficial interactions between host and the oral microbiota urgently require attention. Intriguing questions relate to the acquisition of the oral microbiota in early life, with a possible involvement of pre-natal tolerance development toward the oro-pharyngeal microbiota of the mother. Why do placentas in healthy pregnancies harbor microbiota at all and why does this microbiome resemble the oral communities? Is the fetus already seeded with maternal microbes in utero, as has been proposed recently (Aagaard et al., 2014)? How are these microbiota transported to placenta? Is this transportation selective? Do fetal APCs acquire antigens from placental tissues? If our views on fetal tolerance training by the placental microbiome are confirmed, how is the immune-modulation of the fetus guided by the placental microbiome? Are then vital bacteria involved in recognition by fetal immune system, or just their DNA fragments?

Once the microbiome is established, sustaining it at health becomes an issue. Colonization resistance mechanisms are just becoming apparent and more research needs to be performed using complex model systems and longitudinal clinical studies. Extremely exciting is the overlap between taste and chemical sensing of bacteria in the airways with possible similar mechanisms operating in the mouth. In addition to modulating taste, does the oral microbiota dictate the feeling of hunger or craving for specific dietary habits? Such interactions have been suggested recently where ingestion of bacterial LPS was shown to inhibit taste responses to sucrose in mice (Zhu et al., 2014). Substantial research has been done in the past few years, while one thing is certain-much more research needs to be done in the near future, leading to exciting discoveries directed to maintenance of oral and overall health in an ever-changing population.

\section{ACKNOWLEDGMENTS}

We are thankful to Dr. Kelvin Li from J. Craig Venter Institute for providing the sequences of the most commonly shared oral OTU (OTU\#2) in Human Microbiome Project dataset and to Dr. Bernd Brandt from Department of Preventive Dentistry at ACTA for performing the BLAST search on these sequences. We thank Emil Aritis for drawing the figure. Elena A. Nicu and Bastiaan P. Krom are supported by a grant from the University of Amsterdam for research into the focal point "Oral Infections and Inflammation."

\section{REFERENCES}

Aagaard, K., Ma, J., Antony, K. M., Ganu, R., Petrosino, J., and Versalovic, J. (2014). The placenta harbors a unique microbiome. Sci. Transl. Med. 6:237ra265. doi: 10.1126/scitranslmed.3008599

Allam, J.-P., Peng, W.-M., Appel, T., Wenghoefer, M., Niederhagen, B., Bieber, T., et al. (2008). Toll-like receptor 4 ligation enforces tolerogenic properties of oral mucosal Langerhans cells. J. Allergy Clin. Immunol. 121, 368.e361-374.e361. doi: 10.1016/j.jaci.2007.09.045

Baca, P., Castillo, A. M., Liebana, M. J., Castillo, F., Martin-Platero, A., and Liebana, J. (2012). Horizontal transmission of Streptococcus mutans in schoolchildren. Med. Oral. Patol. Oral. Cir. Bucal. 17, e495-e500. doi: 10.4317/medoral.17592

Bartold, P. M., and Van Dyke, T. E. (2013). Periodontitis: a host-mediated disruption of microbial homeostasis. Unlearning learned concepts. Periodontol. 2000 62, 203-217. doi: 10.1111/j.1600-0757.2012.00450.x

Bearfield, C., Davenport, E. S., Sivapathasundaram, V., and Allaker, R. P. (2002). Possible association between amniotic fluid micro-organism infection and microflora in the mouth. BJOG 109, 527-533. doi: 10.1111/j.14710528.2002.01349.x

Belibasakis, G. N. (2014). Microbiological and immuno-pathological aspects of peri-implant diseases. Arch. Oral. Biol. 59, 66-72. doi: 10.1016/j.archoralbio.2013.09.013

Bradshaw, D. J., and Lynch, R. J. M. (2013). Diet and the microbial aetiology of dental caries: new paradigms. Int. Dent. J. 63, 64-72. doi: 10.1111/idj.12082

Clemente, J. C., Ursell, L. K., Parfrey, L. W., and Knight, R. (2012). The Impact of the gut microbiota on human health: an integrative view. Cell 148, 1258-1270. doi: 10.1016/j.cell.2012.01.035

Costello, E. K., Carlisle, E. M., Bik, E. M., Morowitz, M. J., and Relman, D. A. (2013). Microbiome assembly across multiple body sites in low-birthweight infants. mBio 4, e00782-e00713. doi: 10.1128/mBio.00782-13

Costello, E. K., Lauber, C. L., Hamady, M., Fierer, N., Gordon, J. I., and Knight, R. (2009). Bacterial community variation in human body habitats across space and time. Science 326, 1694-1697. doi: 10.1126/science.1177486

Diaz, P. I., Hong, B.-Y., Frias-Lopez, J., Dupuy, A. K., Angeloni, M., Abusleme, L., et al. (2013). Transplantation-associated long-term immunosuppression promotes oral colonization by potentially opportunistic pathogens without impacting other members of the salivary bacteriome. Clin. Vaccine Immunol. 20, 920-930. doi: 10.1128/CVI.00734-12

Ding, T., and Schloss, P. D. (2014). Dynamics and associations of microbial community types across the human body. Nature 509, 357-360. doi: 10.1038 /nature13178

Dodds, M. W. J., Johnson, D. A., and Yeh, C.-K. (2005). Health benefits of saliva: a review. J. Dent. 33, 223-233. doi: 10.1016/j.jdent.2004.10.009

Dominguez-Bello, M. G., Costello, E. K., Contreras, M., Magris, M., Hidalgo, G., Fierer, N., et al. (2010). Delivery mode shapes the acquisition and structure of the initial microbiota across multiple body habitats in newborns. Proc. Natl. Acad. Sci. U.S.A. 107, 11971-11975. doi: 10.1073/pnas.1002601107

Dupuy, A. K., David, M. S., Li, L., Heider, T. N., Peterson, J. D., Montano, E. A., et al. (2014). Redefining the human oral mycobiome with improved practices in amplicon-based taxonomy: discovery of malassezia as a prominent commensal. PLoS ONE 9:e90899. doi: 10.1371/journal.pone.0090899

Everest-Dass, A. V., Jin, D., Thaysen-Andersen, M., Nevalainen, H., Kolarich, D., and Packer, N. H. (2012). Comparative structural analysis of the glycosylation of salivary and buccal cell proteins: innate protection against infection by Candida albicans. Glycobiology 22, 1465-1479. doi: 10.1093/glycob/cws112

Fardini, Y., Chung, P., Dumm, R., Joshi, N., and Han, Y. W. (2010). Transmission of diverse oral bacteria to murine placenta: evidence for the oral microbiome as a potential source of intrauterine infection. Infect. Immun. 78, 1789-1796. doi: 10.1128/IAI.01395-09

Feller, L., Altini, M., Khammissa, R. A. G., Chandran, R., Bouckaert, M., and Lemmer, J. (2013). Oral mucosal immunity. Oral Surg. Oral Med. Oral Pathol. Oral Radiol. 116, 576-583. doi: 10.1016/j.oooo.2013.07.013

Ghannoum, M. A., Jurevic, R. J., Mukherjee, P. K., Cui, F., Sikaroodi, M., Naqvi, A., et al. (2010). Characterization of the oral fungal microbiome (mycobiome) in healthy individuals. PLoS Pathog. 6:e1000713. doi: 10.1371/journal.ppat. 1000713

Hall, J. A., Bouladoux, N., Sun, C. M., Wohlfert, E. A., Blank, R. B., Zhu, Q., et al. (2008). Commensal DNA dimits regulatory $\mathrm{T}$ cell conversion and is a natural adjuvant of intestinal immune responses. Immunity 29, 637-649. doi: 10.1016/j.immuni.2008.08.009 
He, X., McLean, J. S., Guo, L., Lux, R., and Shi, W. (2013). The social structure of microbial community involved in colonization resistance. ISME J. 8, 564-574. doi: 10.1038/ismej.2013.172

Holgerson, P. L., Vestman, N. R., Claesson, R., Ohman, C., Domellof, M., Tanner, A. C., et al. (2013). Oral microbial profile discriminates breast-fed from formula-fed infants. J. Pediatr. Gastroenterol. Nutr. 56, 127-136. doi: 10.1097/MPG.0b013e31826f2bc6

Hooper, L. V., and Gordon, J. I. (2001). Commensal host-bacterial relationships in the gut. Science 292, 1115-1118. doi: 10.1126/science.1058709

Iliev, I. D., Matteoli, G., and Rescigno, M. (2007). The yin and yang of intestinal epithelial cells in controlling dendritic cell function. J. Exp. Med. 204, 2253-2257. doi: 10.1084/jem.20062535

Janardhanam, S. B., Prakasam, S., Swaminathan, V. T., Kodumudi, K. N., Zunt, S. L., and Srinivasan, M. (2012). Differential expression of TLR-2 and TLR-4 in the epithelial cells in oral lichen planus. Arch. Oral Biol. 57, 495-502. doi: 10.1016/j.archoralbio.2011.10.013

Jarosz, L. M., Ovchinnikova, E. S., Meijler, M. M., and Krom, B. P. (2011). Microbial spy games and host response: roles of a Pseudomonas aeruginosa small molecule in communication with other species. PLoS Pathog. 7:e1002312. doi: 10.1371/journal.ppat.1002312

Jenkinson, H. F. (2011). Beyond the oral microbiome. Environ. Microbiol. 13, 1462-2920. doi: 10.1111/j.1462-2920.2011.02573.x

Jeurink, P. V., van Bergenhenegouwen, J., Jiménez, E., Knippels, L. M. J., Fernández, L., Garssen, J., et al. (2013). Human milk: a source of more life than we imagine. Benef. Microbes 4, 17-30. doi: 10.3920/BM2012.0040

Jiménez, E., Marín, M. L., Martín, R., Odriozola, J. M., Olivares, M., Xaus, J., et al. (2008). Is meconium from healthy newborns actually sterile? Res. Microbiol. 159, 187-193. doi: 10.1016/j.resmic.2007.12.007

Jiménez, E., Fernández, L., Marín, M., Martín, R., Odriozola, J., Nueno-Palop, C., et al. (2005). Isolation of commensal bacteria from umbilical cord blood of healthy neonates born by Cesarean section. Curr. Microbiol. 51, 270-274. doi: 10.1007/s00284-005-0020-3

Khan, S. A., Fidel, P. L. Jr., Thunayyan, A. A., Varlotta, S., Meiller, T. F., and Jabra-Rizk, M. A. (2013). Impaired Histatin-5 levels and salivary antimicrobial activity against in HIV Infected individuals . J. AIDS Clin. Res. 4:1000193. doi: 10.4172/2155-6113.1000193

Kilian, M., Reinholdt, J., Lomholt, H., Poulsen, K., and Frandsen, E. V. (1996). Biological significance of IgAl proteases in bacterial colonization and pathogenesis: critical evaluation of experimental evidence. APMIS 104, 321-338

Krom, B. P., Kidwai, S., and Ten Cate, J. M. (2014). Candida and other fungal species: forgotten players of healthy oral microbiota. J. Dent. Res. 93, 445-451. doi: 10.1177/0022034514521814

Kuramitsu, H. K., He, X., Lux, R., Anderson, M. H., and Shi, W. (2007). Interspecies interactions within oral microbial communities. Microbiol. Mol. Biol. Rev. 71, 653-670. doi: 10.1128/MMBR.00024-07

Layden, B. T., Angueira, A. R., Brodsky, M., Durai, V., and Lowe, W. L. (2013). Short chain fatty acids and their receptors: new metabolic targets. Transl. Res. J. Lab. Clin. Med. 161, 131-140. doi: 10.1016/j.trsl.2012.10.007

Lee, R. J., Kofonow, J. M., Rosen, P. L., Siebert, A. P., Chen, B., Doghramji, L., et al. (2014). Bitter and sweet taste receptors regulate human upper respiratory innate immunity. J. Clin. Invest. 124, 1393-1405. doi: 10.1172/JCI72094

Lee, R. J., Xiong, G., Kofonow, J. M., Chen, B., Lysenko, A., Jiang, P., et al. (2012). T2R38 taste receptor polymorphisms underlie susceptibility to upper respiratory infection. J. Clin. Invest. 122, 4145-4159. doi: 10.1172/JCI64240

Li, K., Bihan, M., and Methe, B. A. (2013). Analyses of the stability and core taxonomic memberships of the human microbiome. PLOS ONE 8:e63139. doi: 10.1371/journal.pone.0063139

Li, Y., Caufield, P. W., Dasanayake, A. P., Wiener, H. W., and Vermund, S. H. (2005). Mode of delivery and other maternal factors influence the acquisition of Streptococcus mutans in infants. J. Dent. Res. 84, 806-811. doi: 10.1177/154405910508400905

Lif Holgerson, P., Harnevik, L., Hernell, O., Tanner, A. C., and Johansson, I. (2011). Mode of birth delivery affects oral microbiota in infants. J. Dent. Res. 90, 1183-1188. doi: 10.1177/0022034511418973

Macpherson, A. J., and Harris, N. L. (2004). Interactions between commensal intestinal bacteria and the immune system. Nat. Rev. Immunol. 4, 478-485. doi: 10.1038/nri1373

Marsh, P. D., Martin, M. V., Lewis, M. A. O., and Williams, D. W. (2009). Oral Microbiology, 5 Edn. Edinburgh: Churchill Livingstone Elsevier.
Mold, J. E., Michaëlsson, J., Burt, T. D., Muench, M. O., Beckerman, K. P., Busch, M. P., et al. (2008). Maternal alloantigens promote the development of tolerogenic fetal regulatory $\mathrm{T}$ cells in utero. Science 322, 1562-1565. doi: 10.1126/science. 1164511

Morales, D. K., and Hogan, D. A. (2010). Candida albicans interactions with bacteria in the context of human health and disease. PLoS Pathog. 6:e1000886. doi: 10.1371/journal.ppat.1000886

Mukherjee, P. K., Chandra, J., Retuerto, M., Sikaroodi, M., Brown, R. E., Jurevic, R., et al. (2014). Oral mycobiome analysis of hiv-infected patients: identification of Pichia as an antagonist of opportunistic fungi. PLoS Pathog. 10:e1003996. doi: 10.1371/journal.ppat.1003996

Niederman, R. (2013). Pregnancy gingivitis and causal inference. Evid. Based Dent. 14, 107-108. doi: 10.1038/sj.ebd.6400966

Novak, N., Haberstok, J., Bieber, T., and Allam, J.-P. (2008). The immune privilege of the oral mucosa. Trends Mol. Med. 14, 191-198. doi: 10.1016/j.molmed.2008. 03.001

Nyvad, B., Crielaard, W., Mira, A., Takahashi, N., and Beighton, D. (2013). Dental caries from a molecular microbiological perspective. Caries Res. 47, 89-102. doi: $10.1159 / 000345367$

Olszak, T., An, D., Zeissig, S., Vera, M. P., Richter, J., Franke, A., et al. (2012). Microbial exposure during early life has persistent effects on natural killer $\mathrm{T}$ cell function. Science 336, 489-493. doi: 10.1126/science.1219328

Palmer, R. J. Jr. (2014). Composition and development of oral bacterial communities. Periodontol. 2000 64, 20-39. doi: 10.1111/j.1600-0757.2012. 00453.x

Petti, S., Polimeni, A., Berloco, P. B., and Scully, C. (2013). Orofacial diseases in solid organ and hematopoietic stem cell transplant recipients. Oral Dis. 19, 18-36. doi: 10.1111/j.1601-0825.2012.01925.x

Prince, A. (2012). The bitter taste of infection. J. Clin. Invest. 122, 3847-3849. doi 10.1172/JCI66182

Rabe, H., Nordström, I., Andersson, K., Lundell, A.-C., and Rudin, A. (2014). Staphylococcus aureus convert neonatal conventional CD4+T cells into FOXP3+CD25+CD127 lowT cells via the PD-1/PD-L1 axis. Immunology 141, 467-481. doi: 10.1111/imm.12209

Rasiah, I. A., Wong, L., Anderson, S. A., and Sissons, C. H. (2005). Variation in bacterial DGGE patterns from human saliva: over time, between individuals and in corresponding dental plaque microcosms. Arch. Oral Biol. 50, 779-787. doi: 10.1016/j.archoralbio.2005.02.001

Romani Vestman, N., Timby, N., Holgerson, P., Kressirer, C., Claesson, R., Domellof, M., et al. (2013). Characterization and in vitro properties of oral lactobacilli in breastfed infants. BMC Microbiol. 13:193. doi: 10.1186/14712180-13-193

Santoro, A., Majorana, A., Roversi, L., Gentili, F., Marrelli, S., Vermi, W., et al. (2005). Recruitment of dendritic cells in oral lichen planus. J. Pathol. 205, 426-434. doi: 10.1002/path.1699

Scholz, F., Badgley, B. D., Sadowsky, M. J., and Kaplan, D. H. (2014). Immune mediated shaping of microflora community composition depends on barrier site. PLoS ONE 9:e84019. doi: 10.1371/journal.pone.0084019

Shetty, V., Pooja, B. L., and Hegde, A. M. (2014). PROP test: prediction of caries risk by genetic taste perception among the visually impaired children. Spec. Care Dent. 34, 34-40. doi: 10.1111/j.1754-4505.2012.00307.x

Soga, Y., Maeda, Y., Ishimaru, F., Tanimoto, M., Maeda, H., Nishimura, F., et al. (2011). Bacterial substitution of coagulase-negative staphylococci for streptococci on the oral mucosa after hematopoietic cell transplantation. Support. Care Cancer 19, 995-1000. doi: 10.1007/s00520-010-0923-9

Stahringer, S. S., Clemente, J. C., Corley, R. P., Hewitt, J., Knights, D., Walters, W. A., et al. (2012). Nurture trumps nature in a longitudinal survey of salivary bacterial communities in twins from early adolescence to early adulthood. Genome Res. 22, 2146-2152. doi: 10.1101/gr.140608.112

Stout, M. J., Conlon, B., Landeau, M., Lee, I., Bower, C., Zhao, Q., et al. (2013). Identification of intracellular bacteria in the basal plate of the human placenta in term and preterm gestations. Am. J. Obstet. Gynecol. 208, 226.e221-226.e227. doi: 10.1016/j.ajog.2013.01.018

Sutmuller, R. P. M., den Brok, M. H. M. G. M., Kramer, M., Bennink, E. J., Toonen, L. W. J., Kullberg, B.-J., et al. (2006). Toll-like receptor 2 controls expansion and function of regulatory T cells. J. Clin. Invest. 116, 485-494. doi: 10.1172/JCI25439

Takahata, Y., Nomura, A., Takada, H., Ohga, S., Furuno, K., Hikino, S., et al. (2004). CD25+CD4+ T cells in human cord blood: an immunoregulatory subset with 
naive phenotype and specific expression of forkhead box p3 (Foxp3) gene. Exp. Hematol. 32, 622-629. doi: 10.1016/j.exphem.2004.03.012

Wade, W. G. (2013). The oral microbiome in health and disease. Pharmacol. Res. 69, 137-143. doi: 10.1016/j.phrs.2012.11.006

Wendell, S., Wang, X., Brown, M., Cooper, M. E., DeSensi, R. S., Weyant, R. J., et al. (2010). Taste genes associated with dental caries. J. Dent. Res. 89, 1198-1202. doi: $10.1177 / 0022034510381502$

Wright, C. J., Burns, L. H., Jack, A. A., Back, C. R., Dutton, L. C., Nobbs, A. H., et al. (2013). Microbial interactions in building of communities. Mol. Oral Microbiol. 28, 83-101. doi: 10.1111/omi.12012

Zhou, Y., Gao, H., Mihindukulasuriya, K. A., La Rosa, P. S., Wylie, K. M., Vishnivetskaya, T., et al. (2013). Biogeography of the ecosystems of the healthy human body. Genome Biol. 14, R1. doi: 10.1186/gb-2013-14-1-r1

Zhu, X., He, L., and McCluskey, L. P. (2014). Ingestion of bacterial lipopolysaccharide inhibits peripheral taste responses to sucrose in mice. Neuroscience 258, 47-61. doi: 10.1016/j.neuroscience.2013.10.072
Conflict of Interest Statement: The authors declare that the research was conducted in the absence of any commercial or financial relationships that could be construed as a potential conflict of interest.

Received: 01 April 2014; accepted: 08 June 2014; published online: 26 June 2014. Citation: Zaura E, Nicu EA, Krom BP and Keijser BJF (2014) Acquiring and maintaining a normal oral microbiome: current perspective. Front. Cell. Infect. Microbiol. 4:85. doi: 10.3389/fcimb.2014.00085

This article was submitted to the journal Frontiers in Cellular and Infection Microbiology.

Copyright (ㅇ 2014 Zaura, Nicu, Krom and Keijser. This is an open-access article distributed under the terms of the Creative Commons Attribution License (CC BY). The use, distribution or reproduction in other forums is permitted, provided the original author(s) or licensor are credited and that the original publication in this journal is cited, in accordance with accepted academic practice. No use, distribution or reproduction is permitted which does not comply with these terms. 